Review Article

\title{
Hyperuricemia in Children and Adolescents: Present Knowledge and Future Directions
}

\author{
Masaru Kubota \\ Department of Agriculture, Ryukoku University, Ohtsu, Shiga, Japan \\ Correspondence should be addressed to Masaru Kubota; masaru_kubota@chime.ocn.ne.jp
}

Received 6 February 2019; Accepted 9 April 2019; Published 2 May 2019

Academic Editor: José María Huerta

Copyright (c) 2019 Masaru Kubota. This is an open access article distributed under the Creative Commons Attribution License, which permits unrestricted use, distribution, and reproduction in any medium, provided the original work is properly cited.

\begin{abstract}
Recent evidence suggests that hyperuricemia is an important condition in children and adolescents, particularly in association with noncommunicable diseases. This review aims to summarize our current understanding of this condition in pediatric patients. An analysis of serum uric acid reference values in a healthy population indicates that they increase gradually with age until adolescence, with differences between the sexes arising at about 12 years of age. This information should be taken into consideration when defining hyperuricemia in studies. Gout is extremely rare in children and adolescents, and most patients with gout have an underlying disease. The major causes of hyperuricemia are chronic conditions, including Down syndrome, metabolic or genetic disease, and congenital heart disease, and acute conditions, including gastroenteritis, bronchial asthma (hypoxia), malignant disorders, and drug side effects. The mechanisms underlying the associations between these diseases and hyperuricemia are discussed, together with recent genetic information. Obesity is a major cause of hyperuricemia in otherwise healthy children and adolescents. Obesity is often accompanied by metabolic syndrome; hyperuricemia in obese children and adolescents is associated with the components of metabolic syndrome and noncommunicable diseases, including hypertension, insulin resistance, dyslipidemia, and chronic kidney disease. Finally, strategies for the treatment of hyperuricemia, including lifestyle intervention and drug administration, are presented.
\end{abstract}

\section{Introduction}

Hyperuricemia is a laboratory abnormality often observed in children and adolescents. However, because of the low diagnostic value of serum uric acid (hereafter described as "uric acid") alone, uric acid levels may not be adequately considered by pediatricians. More attention has been drawn to hyperuricemia in children and adolescents by several recent studies reporting its association with obesity and noncommunicable diseases (NCDs), especially cardiovascular disorders. A search of the literature found only two reviews of hyperuricemia in children and adolescents $[1,2]$. Although these reviews are comprehensive and well summarized, they have two major drawbacks. First, they do not adequately address the associations between hyperuricemia and NCDs. Second, recent findings of genetic studies on hyperuricemia are not fully discussed. Therefore, this review aims to cover these topics and proposes future directions for research on hyperuricemia in children and adolescents.

\section{Database}

Articles published from 2000 to 2018 were retrieved in searches of Medline and Web of Science. The search methodology consisted of both controlled vocabulary as in the National Library of Medicine MeSH and the keywords uric acid/hyperuricemia and child/adolescent. Reviews and case reports published before 2000 are included if considered to be of particular importance.

\section{Reference Values of Uric Acid in Children and Adolescents}

In adults, serum uric acid $>7.0 \mathrm{mg} / \mathrm{dL}$ is widely used as the definition of hyperuricemia, considering the solubility of uric acid $[3,4]$. However, uric acid levels in children and adolescents change during development. Therefore, age- and sex-related reference values for uric acid should be considered for defining hyperuricemia in children and 
adolescents. Summarizing previous reports, the following developmental changes in uric acid levels have been identified although the absolute uric acid values differ marginally from report to report [1,5-7]. Uric acid levels increase gradually from birth to the end of elementary school age. Subsequently, levels rise sharply in males and slightly in females, creating a significant difference between the sexes. For reference, data from two studies $[1,6]$ are shown in Table 1.

\section{Conditions Causing Hyperuricemia}

4.1. Gout. Large-scale epidemiological studies of gout in children and adolescents are quite limited. In a study using data in the UK General Practice Research Database (1990-1999), Mikuls et al. report that the incidence of gout in individuals <25 years was 12 in 255,950 men and 1 in 246,346 women [8]. The incidence between 2007 and 2015 in Korea was 2 to 3 in 100,000 persons aged $0-9$ years and 9 to 20 in 100,000 persons aged 10-19 years [9]. Our nationwide questionnaire survey of pediatric departments identified only seven cases of gout in more than 2,300,000 inpatients and outpatients under 15 years of age [10]. All patients with gout in our study were found to have at least one underlying disorder. Previous case reports have shown that gout in children or adolescents was associated with comorbidities such as Down syndrome [11], glycogen storage disease [12], renal transplantation [13], leukemia [14], and methyl malonic acidemia [15] (Table 2).

\subsection{Chronic Disease}

4.2.1. Metabolic Diseases. Abnormalities in the metabolism of purine or related compounds have been shown to cause hyperuricemia in infancy or childhood $[16,17]$. These abnormalities include complete hypoxanthine-guanine phosphoribosyl transferase (HGPRT) deficiency (Lesch-Nyhan syndrome), partial HGPRT deficiency (Kelly-Seegmiller syndrome), adenine phosphoribosyl transferase (APRT) deficiency, phosphoribosyl pyrophosphate (PRPP) synthetase overactivity, and myoadenylate deaminase deficiency. The most common cause of hyperuricemia is the overproduction of uric acid [1, 17]. Some patients with Lesch-Nyhan syndrome, Kelly-Seegmiller syndrome, or PRPP synthetase overactivity exhibit gout in early childhood or adolescence [1]. Glycogen storage diseases, especially type I, III, V, and VII, also cause hyperuricemia. Muscle exertion in these diseases is thought to result in accelerated degradation of muscle purine nucleotides [1].

4.2.2. Down Syndrome. Down syndrome, seen in approximately one case per $800-1000$ live births, is the most common chromosomal abnormality. The association between Down syndrome and hyperuricemia was described quite early [18]. Since then, several reports have indicated the higher prevalence of hyperuricemia in patients with Down syndrome $[19,20]$. Particularly, Kashima et al. report that hyperuricemia in Down syndrome appears in early
TABLE 1: Reference values of uric acid in children and adolescents.

\begin{tabular}{|c|c|c|c|c|}
\hline & \multicolumn{4}{|c|}{ Uric acid $(\mathrm{mg} / \mathrm{dL})$} \\
\hline & $\operatorname{Sex}^{* *}$ & Age (years) & Mean & SD \\
\hline \multirow{8}{*}{ Wilcox $[1]^{*}$} & & $<5$ & 3.6 & 0.9 \\
\hline & & $5 \sim 10$ & 4.1 & 1.0 \\
\hline & & 12 & 4.4 & 1.1 \\
\hline & Male & 15 & 5.6 & 1.1 \\
\hline & & 18 & 6.2 & 0.8 \\
\hline & & 12 & 4.5 & 0.9 \\
\hline & Female & 15 & 4.5 & 0.9 \\
\hline & & 18 & 4.0 & 0.7 \\
\hline \multirow{8}{*}{ Kubota [6] } & & $<1$ & 2.9 & 0.9 \\
\hline & & $1 \sim 3$ & 3.3 & 0.8 \\
\hline & & $4 \sim 6$ & 3.6 & 1.0 \\
\hline & & $7 \sim 9$ & 4.2 & 0.9 \\
\hline & Male & $10 \sim 12$ & 4.5 & 0.9 \\
\hline & & $>13$ & 5.6 & 1.0 \\
\hline & Female & $10 \sim 12$ & 4.1 & 0.8 \\
\hline & & $>13$ & 4.3 & 0.9 \\
\hline
\end{tabular}

${ }^{*}$ The number within brackets indicate the reference number. ${ }^{* *}$ The blanks in this column indicate both sexes.

infancy, according to their age-specific reference values [20]. The underlying cause of this association is proposed to be elevated levels of purine metabolizing enzymes [21] and lifestyle related factors such as obesity or low physical activity [20]. Recently, Garlet et al. presented the hypothesis that the elevated uric acid level is a compensatory response to the redox imbalance accompanying Down syndrome [22].

4.2.3. Congenital Heart Diseases. Congenital heart diseases, especially cyanotic heart diseases, are often associated with hyperuricemia $[6,23]$. Polycythemia, chronic hypoxia, and the resultant increased purine catabolism are proposed to be the primary pathogenetic mechanisms underlying this association [1]. Rodríguez-Hemández et al. recently postulated that BMI, impaired renal function, cyanosis, and the use of diuretics are risk factors for hyperuricemia in patients with congenital heart diseases [24].

4.2.4. Genetic Disorders. Familial juvenile hyperuricemic nephropathy (FJHN) is characterized by juvenile onset of hyperuricemia, gout, and progressive nephropathy [25]. Hart et al. first determined that a mutation in the uromodulin gene is responsible for FJHN [26]. A genome-wide association study revealed that single-nucleotide polymorphisms in the uric acid transporter genes (ABCG2 and SLC2A9) cause hyperuricemia and gout by altering urinary uric acid clearance [27]. Apolipoprotein E gene polymorphisms are also associated with primary hyperuricemia, as identified by $\mathrm{Wu}$ et al. in a Chinese pediatric population of 770 subjects [28].

\subsection{Acute Diseases}

4.3.1. Gastroenteritis. Our investigation of conditions associated with hyperuricemia in more than 9000 pediatric 
TABLE 2: Diseases/disorders causing hyperuricemia in children and adolescents.

1. Gout

2. Chronic diseases

(a) Metabolic disease

Hypoxanthine-guanine phosphoribosyl transferase

(HGPRT) deficiency

(complete, Lesch-Nyhan syndrome; partial,

Kelly-Seegmiller syndrome)

Adenine phosphoribosyl transferase (APRT) deficiency

Phosphoribosylpyrophosphate (PRPP) synthetase

overactivity

Myoadenylate deaminase deficiency

Glycogen storage diseases (types I, III, V, and VII)

Acyl-coenzyme A dehydrogenase deficiency

(b) Down syndrome

(c) Congenital heart disease (especially cyanotic diseases)

(d) Genetic diseases

Familial juvenile hyperuricemic nephropathy (FJHN)

3. Acute diseases

(a) Gastroenteritis (especially Rotavirus infection)

(b) Bronchial asthma (especially on attacks)

(c) Malignant disorders (tumor lysis syndrome)

(d) Hemolytic anemia

(e) Drugs

Diuretics (thiazide)

Theophylline

Anticonvulsants (valproate and phenobarbital)

Cyclosporine

Pyrazinamide

4. Lifestyle-related disorders

(a) Obesity

(b) Metabolic syndrome

inpatients revealed that gastroenteritis is the most common [6]. Of the agents causing gastroenteritis, rotavirus infection is most likely to cause hyperuricemia $[29,30]$. Dehydration is the suspected cause of hyperuricemia in gastroenteritis; uric acid levels return to normal levels readily after hydration treatment, without the use of antihyperuricemia drugs. In addition, Matsuo et al. suggest an additional possible mechanism involving decreased intestinal uric acid excretion via ABCG2, caused by gastroenteritis-induced injury of the intestinal epithelium [31].

4.3.2. Bronchial Asthma (Hypoxia). Bronchial asthma, especially during an acute attack, is a common cause of hyperuricemia in pediatric patients [6]. Abdulnaby et al. demonstrated that the degree of serum uric acid elevation may serve as a useful marker of the severity of bronchial asthma [32]. Hypoxia and dehydration are considered possible underlying causes of hyperuricemia in bronchial asthma. In a study of sleep-disordered breathing in obese children and adolescents, the degree of arterial oxygen saturation was found to correlate with uric acid levels [33].

4.3.3. Malignant and Hematological Disorders. Pediatric patients with malignant disorders, particularly hematological diseases such as leukemia and lymphoma, are at risk for hyperuricemia $[34,35]$. This risk may be due in part to the large tumor burden at onset characteristics of pediatric malignancies [35]. In addition, the higher susceptibility of children to chemotherapeutic agents places them at greater risk than adult patients for tumor lysis syndrome (TLS) [36].

Hemolytic anemia, such as hemolytic uremic syndrome [37] and sickle cell anemia [38], causes hyperuricemia. Erythrocyte overdestruction and hyperlacticacidemia may play a role in the occurrence of hyperuricemia. Notably, uric acid levels sometimes exceeded $20 \mathrm{mg} / \mathrm{dL}$, necessitating peritoneal dialysis in patients with hemolytic uremic syndrome [37].

4.3.4. Side Effects of Drugs. Several drugs such as diuretics (thiazide), anticonvulsants (valproate and phenobarbital), cyclosporine, theophylline, and pyrazinamide have been reported to increase uric acid levels in children and adolescents $[1,13,39-41]$. Although the underlying causes are not fully understood, renal tubular reabsorption of uric acid, dehydration, and increased purine catabolism has been postulated $[1,42]$.

\subsection{Lifestyle-Related Disorders}

4.4.1. Obesity. Obesity in children and adolescents is the global problem seen in both developed and developing countries [43, 44]. Results of large-scale studies on the prevalence of hyperuricemia in a general population of children and adolescents are summarized in Table 3 [45-49]. Generally, the prevalence of hyperuricemia tends to be higher in Western countries than in Asian countries and in males than in females. The limited reports in an obese population demonstrated a prevalence of several folds higher that in a general population; however, these reports had drawbacks, including smaller cohorts of obese than general populations and heterogeneity in the definition of obesity [50-53]. Furthermore, the mean uric acid levels turn out to be higher in an obese population $[54,55]$. During over a 6-year follow-up period in children aged 6-12 years at study commencement, Kuwahara et al. showed that an excessive increase in BMI during that time was associated with significant uric acid elevation by early adolescence [56].

4.4.2. Metabolic Syndrome (MS). The association of hyperuricemia with MS is well documented in adults [57]. In a recent review of MS in children and adolescents, Bussler et al. demonstrated a prevalence of MS ranging from 6 to 39\%, depending on the applied definition criteria [58]. They also identified hyperuricemia as an important comorbidity, together with nonalcoholic fatty liver disease and sleep restriction. In a cohort of 1370 adolescents aged 12-17 years divided into four quartiles based on the uric acid level, Ford et al. observed a sequentially higher prevalence of MS from the lowest quartile $(<1 \%)$ to the highest quartile $(21.1 \%)$ [45]. In 2284 Taiwanese children aged $6-12$ years, uric acid levels were found to be a significant predictor of MS, exhibiting a $54 \%$ increased risk for MS for every $1 \mathrm{mg} / \mathrm{dL}$ increase in uric acid concentration [46]. The positive association of uric acid levels 
TABle 3: Prevalence of hyperuricemia in a general or obese children and adolescents.

\begin{tabular}{|c|c|c|c|c|c|c|}
\hline Author & Study year & Number & Age (years) & Sex & Uric acid (mg/dL) & Hyperuricemia (\%) \\
\hline \multicolumn{7}{|l|}{ General population } \\
\hline & \multirow{3}{*}{$1999-2002$} & \multirow{3}{*}{1370} & \multirow{3}{*}{$12 \sim 17$} & \multirow{3}{*}{ Both } & $\geqq 5.5$ & 30.2 \\
\hline \multirow[t]{2}{*}{ Ford et al. [45] ${ }^{*}$} & & & & & $\geqq 6.0$ & 22.2 \\
\hline & & & & & $>7.0$ & 6.5 \\
\hline \multirow[t]{2}{*}{ Lee et al. [46] } & \multirow[t]{2}{*}{$2001-2002$} & \multirow[t]{2}{*}{2284} & \multirow[t]{2}{*}{$6 \sim 12$} & Male & $\geqq 7.0$ & 26.5 \\
\hline & & & & Female & & 18.8 \\
\hline \multirow[t]{2}{*}{ Shatat et al. [47] } & \multirow[t]{2}{*}{$2005-2008$} & \multirow[t]{2}{*}{1912} & \multirow[t]{2}{*}{$13 \sim 18$} & Both & $>6.0$ & 19.3 \\
\hline & & & & Male & $\geqq 7.1$ & 5.4 \\
\hline \multirow{3}{*}{ Kawasaki et al. [48] } & \multirow{3}{*}{ 2011-2012 } & \multirow{3}{*}{29714} & \multirow{3}{*}{$<15$} & & $\geqq 8.0$ & 1.6 \\
\hline & & & & Female & $\geqq 7.1$ & 0.45 \\
\hline & & & & & $\geqq 8.0$ & 0.15 \\
\hline \multirow{2}{*}{ Li et al. [49] } & \multirow{2}{*}{2015} & \multirow{2}{*}{4073} & \multirow{2}{*}{$3 \sim 6$} & Male & $\geqq 5.1$ & 11.8 \\
\hline & & & & Female & & 8.3 \\
\hline \multicolumn{7}{|l|}{ Obese population } \\
\hline \multirow{2}{*}{ Tang et al. [50] } & \multirow{2}{*}{$2005-2008$} & \multirow{2}{*}{1027} & \multirow{2}{*}{$6 \sim 14$} & Male & $\geqq 5.9 \sim 7.0$ & 24.4 \\
\hline & & & & Female & $\geqq 5.9 \sim 6.2$ & 15.2 \\
\hline Modino et al. [51] & Not shown & 148 & $5 \sim 19$ & Both & $\geqq 5.5$ & 53 \\
\hline Cardoso et al. [52] & 2009-2010 & 129 & $<18$ & Both & $>5.5$ & 12.4 \\
\hline Ságodi et al. [53] & Not shown & 162 & $10 \sim 14$ & Both & Unknown & 38.3 \\
\hline
\end{tabular}

*The number within brackets indicate the reference number.

or hyperuricemia with the occurrence of MS has been reported in multiple countries, including Japan [50], Spain [51], and Brazil [52]. In addition, several studies report that the relationship between uric acid levels and MS in adolescents is dependent upon racial/ethnic and sex differences $[59,60]$.

\subsection{Components of MS or NCDs}

4.5.1. Hypertension. Hypertension in relation to hyperuricemia has been most extensively investigated among components of MS or NCDs. In a cohort of 125 children aged 6-18 years being evaluated for hypertension, Feig and Johnson observed that uric acid levels correlate directly with blood pressure in untreated children and that a concentration $>5.5 \mathrm{mg} / \mathrm{dL}$ in adolescents strongly suggest primary hypertension [61]. Numerous studies in different countries have subsequently confirmed this association between hyperuricemia and hypertension in children and adolescents $[49,55,62,63]$. In the tracking of 449 children aged 3-7, Park et al. found that children with high uric acid levels at both 3 and 5 years had the highest systolic blood pressure at 7 years [64]. In addition, by the average follow-up period of 12 years, elevated childhood uric acid levels are shown to be associated with increased blood pressure in beginning in children and higher blood pressure levels that persist into adulthood [65]. Taking these reports into consideration, Yanik and Feig suggest the use of the uric acid level as a possible biomarker for diagnosing essential hypertension in children [66].

4.5.2. Insulin Resistance. In an earlier study of Japanese obese children aged 7-15 years, fasting insulin levels correlated positively with uric acid levels [67]. After adjustment by age, sex, and BMI, uric acid levels were found to be positively associated with insulin resistance as evaluated by homeostasis model assessment (HOMA-R) and negatively associated with adiponectin concentration [54]. A study of Greek obese youths indicates an association between hyperuricemia and HOMA-R [68].

4.5.3. Dyslipidemia and Atherosclerosis. Denzer et al. observed that triglycerides and the cholesterol/HDL ratio correlated positively with uric acid levels in a cohort of 269 obese children whose BMI was $>90^{\text {th }}$ percentile [69]. In a study of different ethnic groups in Taiwan, hypertriglycemia and hypercholesterolemia were associated with hyperuricemia, but the tendency differed between ethnic groups (Aborigines vs. non-Aborigines) [70]. Hyperlipidemia is associated with an increased risk of atherosclerosis in children and adolescents [71]. Pacifico et al. showed that the carotid intima-medial thickness, an indicator of atherosclerosis, was elevated in participants in the fourth quartile of uric acid compared to those in the first, second, and third quartiles [72]. Furthermore, in Japanese obese children, uric acid levels were shown to correlate positively with lipids and negatively with flow-mediated dilatation of the brachial artery [73]. Together, these results suggest that hyperuricemia in obese children may be a marker for early atherosclerosis.

4.5.4. Chronic Kidney Disease (CKD). A recent review suggests that uric acid plays a role in the pathogenesis of CKD in children [74]. Rodenbach et al. demonstrated that hyperuricemia was an independent risk factor for faster progression of CKD in a cohort of over 600 children and adolescents over a 5-year period [75]. Furthermore, lowering of uric acid levels with allopurinol over a 4-month period improved eGFR independently in children with stage 1-3 CKD [76]. 


\section{Treatment of Hyperuricemia}

Apart from a causal relationship, it is clear that hyperuricemia and those four components of NCDs described above are closely associated each other. Hyperuricemia in children and adolescents is a target of treatment, considering the report demonstrating that pediatric patients with hyperuricemia are at increased risk of mortality, particularly due to kidney and cardiovascular diseases [77].

5.1. Lifestyle Intervention. Since obesity is a major cause of hyperuricemia in otherwise healthy children and adolescents, programs for reducing body weight by lifestyle intervention (dietary, physical activity, and behavioral changes) are important [78]. To my best knowledge, however, only two studies addressing this issue are present in the literature. Togashi et al. demonstrated that uric acid levels decreased significantly in 33 obese children after diet plus exercise treatment for 3 months [79]. Furthermore, a oneyear weight reduction program in a cohort of 10-17-yearolds was reported to decrease uric levels in $86 \%$ of the females and $67 \%$ of the males [80]. Although the effect of programs of intervening a lifestyle on obesity is promising, the effect on hyperuricemia requires further investigation over a longer observation period.

5.2. Xanthine Oxidase Inhibitors. Allopurinol, an inhibitor of xanthine oxidase, is an old drug commonly used to treat a variety of pediatric diseases $[17,25]$, including HGPRT deficiency [81], APRT deficiency [82], glycogen storage disease type Ia [83], and FJHN [84]. In addition, the efficacy of allopurinol treatment alone [85] or in combination with enalapril [86] in reducing blood pressure has been investigated in children with hyperuricemic hypertension. However, allopurinol should be used with caution as it can cause severe skin side effects, including Stevens-Johnson syndrome (SJS). The association of allopurinol-induced SJS with human leukocyte antigen (HLA)-B $* 5801$ has been identified [87].

Febuxostat is a newly developed nonpurine, selective inhibitor of xanthine oxidase [88]. Kaku and Nishimura administered febuxostat to 16 children with CKD and observed a renal protective effect accompanied by the lowering of uric acid levels [89]. When used as prophylaxis for TLS in pediatric hematological malignancies, an effect comparable to that of allopurinol was observed [90]. Further investigation is required to determine the efficacy and safety of febuxostat for use in children and adolescents.

5.3. Uric Acid Oxidase (Rasburicase). A recombinant uric acid oxidase, rasburicase, is widely used for the prevention of hyperuricemia observed in TLS at diagnosis or during treatment of a variety of malignancies in children [91, 92]. Rasburicase treatment achieved a greater and more rapid decline in uric acid levels than did allopurinol [91]. Despite the efficacy of rasburicase in malignancy-induced TLS, Cheuk et al. raised concerns about its serious side effects, including hypersensitivity and hemolysis [93].

\section{Conclusion and Future Directions}

This review raises three points that should be considered to improve and shape the direction of future research on hyperuricemia in children and adolescents. First, the reference values of uric acid in children and adolescents change with age, with a difference between the sexes arising at about 12 years of age. Therefore, the definition of hyperuricemia used in data analysis should take these factors into account. Second, future studies should address the question of how hyperuricemia arising in childhood or adolescence affects health in adulthood, especially regarding NCDs. Large cohort, long-term follow-up studies are needed to answer this question. Third, the treatment of hyperuricemia in children and adolescents should be investigated with the aim of standardization, including recommendations as to when uric acid-lowering treatment should be initiated and which drugs are most suitable. These factors are particularly important with respect to chronic diseases that cause hyperuricemia starting early in childhood. The efficacy of treatment through lifestyle intervention also should be investigated. As our understanding of the importance of hyperuricemia in childhood improves, pediatricians should pay greater attention to hyperuricemia in the clinical setting.

\section{Disclosure}

The work described has not been published before and is not under consideration by any other journals.

\section{Conflicts of Interest}

The author declares that there are no conflicts of interest.

\section{Acknowledgments}

The author would like to thank Enago (www.enago.jp) for the English language reviews.

\section{References}

[1] W. D. Wilcox, "Abnormal serum uric acid levels in children," Journal of Pediatrics, vol. 128, no. 6, pp. 731-741, 1996.

[2] H. Yamanaka, "Gout and hyperuricemia in young people," Current Opinion in Rheumatology, vol. 23, no. 2, pp. 156-160, 2011.

[3] T. Neogi, "Gout," New England Journal of Medicine, vol. 364, no. 5, pp. 443-452, 2011.

[4] H. Yamanaka and Japanese Society of Gout and Nucleic Acid Metabolism, "Japanese guideline for the management of hyperuricemia and gout: second edition," Nucleosides, Nucleotides Nucleic Acids, vol. 30, no. 12, pp. 1018-1029, 2011.

[5] S. M. Clifford, A. M. Bunker, J. R. Jacobsen, and W. L. Roberts, "Age and gender specific pediatric reference intervals for aldolase, amylase, ceruloplasmin, creatine kinase, pancreatic amylase, prealbumin, and uric acid," Clinica Chimica Acta, vol. 412, no. 9-10, pp. 788-790, 2011.

[6] M. Kubota, A. Nagai, L. Tang, and M. Tokuda, "Investigation on hyperuricemia in children with obesity or various pediatric disorders," Nucleosides, Nucleotides and Nucleic Acids, vol. 30, no. 12, pp. 1051-1059, 2011. 
[7] T. P. Loh and M. P. Metz, "Trends and physiology of common serum biochemistries in children aged 0-18 years," Pathology, vol. 47, no. 5, pp. 452-461, 2015.

[8] T. R. Mikuls, J. T. Farrar, W. B. Bilker, S. Fernandes, H. R. Schumacher Jr., and K. G. Saag, "Gout epidemiology: results from the UK general practice research database, 1990-1999," Annals of the Rheumatic Diseases, vol. 64, no. 2, pp. 267-272, 2005.

[9] J.-W. Kim, S. G. Kwak, H. Lee, S.-K. Kim, J.-Y. Choe, and S.-H. Park, "Prevalence and incidence of gout in Korea: data from the national health claims database 2007-2015," Rheumatology International, vol. 37, no. 9, pp. 1499-1506, 2017.

[10] R. Kato, M. Kubota, Y. Higashiyama, and A. Nagai, "A nationwide epidemiological study of gout in childhood and adolescence," Gout and Nucleic Acid Metabolism, vol. 38, no. 1, pp. 43-48, 2014, in Japanese.

[11] M. L. Ciompi, L. M. Bazzichi, D. Bertolucci et al., "Uric acid metabolism in two patients with coexistent Down's syndrome and gout," Clinical Rheumatology, vol. 3, no. 2, pp. 229-233, 1984.

[12] W. Zhang, C.-d. Bao, Y.-y. Gu, and S. Ye, "Glycogen storage disease manifested as gout and myopathy: three case reports and literature review," Clinical Rheumatology, vol. 27, no. 5, pp. 671-674, 2008.

[13] J. Trück, G. F. Laube, R. O. von Vigier, and P. Goetschel, "Gout in pediatric renal transplant recipients," Pediatric Nephrology, vol. 25, no. 12, pp. 2535-2538, 2010.

[14] C. J. Morley, I. B. Houston, and P. Morris-Jones, “Acute renal failure and gout as presenting features of acute lymphoblastic leukaemia," Archives of Disease in Childhood, vol. 51, no. 9, pp. 723-725, 1976.

[15] S. Charuvanij, A. Pattaragarn, W. Wisuthsarewong, and N. Vatanavicharn, "Juvenile gout in methylmalonic acidemia," Pediatrics International, vol. 58, no. 6, pp. 501-503, 2016.

[16] J. S. Cameron, F. Moro, and H. A. Simmonds, "Gout, uric acid and purine metabolism in paediatric nephrology," Pediatric Nephrology, vol. 7, no. 1, pp. 105-118, 1993.

[17] W. L. Nyhan, Inherited Hyperuricemic Disorders, Contributions to Nephrology, vol. 147, 2005.

[18] R. W. Fuller, M. W. Luce, and E. T. Mertz, "Serum uric acid in mongolism," Science, vol. 137, no. 3533, pp. 868-869, 1962.

[19] S. Málaga, R. Pardo, I. Málaga, G. Orejas, and J. FernándezToral, "Renal involvement in Down syndrome," Pediatric Nephrology, vol. 20, no. 5, pp. 614-617, 2005.

[20] A. Kashima, Y. Higashiyama, M. Kubota, C. Kawaguchi, Y. Takahashi, and T. Nishikubo, "Children with Down's syndrome display high rates of hyperuricaemia," Acta Paediatrica, vol. 103, no. 8, pp. e359-e364, 2014.

[21] R. Puukka, M. Puukka, L. Perkkila, and K. Kouvalainen, "Levels of some purine metabolizing enzymes in lymphocytes from patients with Down's syndrome," Biochemical Medicine and Metabolic Biology, vol. 36, no. 1, pp. 45-50, 1986.

[22] T. R. Gartlet, E. B. Parisotto, S. de Medeiros Gda et al., "Systemic oxidative stress in children and teenagers with Down syndrome," Life Sciences, vol. 93, no. 16, pp. 558-563, 2013.

[23] E. Martínez-Quintana and F. Rodríguez-González, "Hyperuricaemia in congenital heart disease patients," Cardiology in the Young, vol. 25, no. 1, pp. 29-34, 2015.

[24] J. L. Rodríguez-Hemández, F. Rodríguez-González, M. RiañoRuiz, and E. Martínez-Quintana, "Risk factors for hyperuricemia in congenital heart disease patients and its relation to cardiovascular death," Congenital Heart Disease, vol. 13, no. 5, pp. 655-662, 2018.

[25] J. S. Cameron and H. A. Simmonds, "Hereditary hyperuricemia and renal disease," Seminars in Nephrology, vol. 25, no. 1, pp. 9-18, 2005.

[26] T. C. Hart, M. C. Gorry, P. S. Hart et al., "Mutations of the UMOD gene are responsible for medullary cystic kidney disease 2 and familial juvenile hyperuricaemic nephropathy," Journal of Medical Genetics, vol. 39, no. 12, pp. 882-892, 2002.

[27] H. Matsuo, K. Yamamoto, H. Nakaoka et al., "Genome-wide association study of clinically defined gout identifies multiple risk loci and its association with clinical subtypes," Annals of the Rheumatic Diseases, vol. 75, no. 4, pp. 652-659, 2016.

[28] J. Wu, L. Qiu, X. Z. Guo et al., "Apolipoprotein E gene polymorphisms are associated with primary hyperuricemia in a Chinese population," PLoS One, vol. 9, no. 10, Article ID e110864, 2014.

[29] A. Kovacs, L. Chan, C. Hotrakitya, G. Overturf, and B. Portnoy, "Rotavirus gastroenteritis," American Journal of Diseases of Children, vol. 141, no. 2, pp. 161-166, 1987.

[30] E. Palumbo, M. Branchi, C. Malorgio, A. Siani, and G. Bonora, "Diarrhea in children: etiology and clinical aspects," Minerva pediatrica, vol. 62, no. 4, pp. 347-351, 2010.

[31] H. Matsuo, T. Tsunoda, K. Ooyama et al., "Hyperuricemia in acute gastroenteritis is caused by decreased urate excretion via ABCG2," Scientific Reports, vol. 6, no. 1, p. 31003, 2016.

[32] N. K. Abdulnaby, A. O. Sayed, and N. M. Shalaby, "Predictive value of serum uric acid in hospitalized adolescents and adults with acute asthma," Therapeutics and Clinical Risk Management, vol. 12, pp. 1701-1708, 2016.

[33] S. L. Verhulst, K. Van Hoeck, N. Schrauwen et al., "Sleepdisordered breathing and uric acid in overweight and obese children and adolescents," Chest, vol. 132, no. 1, pp. 76-80, 2007.

[34] D. P. Jones, F. B. Stapleton, D. Kalwinsky, C. P. McKay, S. J. Kellie, and C.-H. Pui, "Renal dysfunction and hyperuricemia at presentation and relapse of acute lymphoblastic leukemia," Medical and Pediatric Oncology, vol. 18, no. 4, pp. 283-286, 1990.

[35] A. Nagai, M. Kubota, L. Tang, S. Adachi, I. Usami, and K. Matsubara, "Hyperuricemia in pediatric malignancies before treatment," Nucleosides, Nucleotides and Nucleic Acids, vol. 30, no. 12, pp. 1060-1065, 2011.

[36] P. J. Galardy, J. Hochberg, S. L. Perkins, L. Harrison, S. Goldman, and M. S. Cairo, "Rasburicase in the prevention of laboratory/clinical tumour lysis syndrome in children with advanced mature B-NHL: a children's oncology group report," British Journal of Haematology, vol. 163, no. 3, pp. 365-372, 2013.

[37] B. S. Kaplan and P. D. Thomson, "Hyperuricemia in the hemolytic-uremic syndrome," Archives of Pediatrics \& Adolescent Medicine, vol. 130, no. 8, pp. 854-856, 1976.

[38] H. S. Diamond, A. D. Meisel, and D. Holden, "The natural history of urate overproduction in sickle cell anemia," Annals of Internal Medicine, vol. 90, no. 5, pp. 752-757, 1979.

[39] T. Shimizu, A. Morikawa, S. Maeda, H. Mochizuki, K. Tokuyama, and T. Kuroume, "Effect of theophylline on serum uric acid levels in children with asthma," Journal of Asthma, vol. 31, no. 5, pp. 387-391, 1994.

[40] H. A. Ring, A. J. Heller, W. J. Marshall, A. L. Johnson, and E. H. Reynolds, "Plasma uric acid in patients receiving anticonvulsant monotherapy," Epilepsy Research, vol. 8, no. 3, pp. 241-244, 1991. 
[41] T. Şişmanlar, A. T. Aslan, and I. Budakoğlu, "Is hyperuricemia overlooked when treating pediatric tuberculosis patients with pyrazinamide?," Journal of Tropical Pediatrics, vol. 61, no. 5, pp. 351-356, 2015.

[42] T. Yamamoto, Y. Moriwaki, M. Suda, S. Takahashi, K. Hiroishi, and K. Higashino, "Theophylline-induced increase in plasma uric acid-purine catabolism increased by theophylline," International Journal of Clinical Pharmacology, Therapy, and Toxicology, vol. 29, no. 7, pp. 257-261, 1991.

[43] Y. Wang and H. Lim, "The global childhood obesity epidemic and the association between socio-economic status and childhood obesity," International Review of Psychiatry, vol. 24, no. 3, pp. 176-188, 2012.

[44] M. Kubota and A. Nagai, "Factors associated with childhood obesity in Asian countries: a review of recent literature," in Recent Advances in Obesity in Children (Chapter 2), Avid Science, Hyderabad, India, 2015.

[45] E. S. Ford, C. Li, S. Cook, and H. K. Choi, "Serum concentrations of uric acid and the metabolic syndrome among US children and adolescents," Circulation, vol. 115, no. 19, pp. 2526-2532, 2007.

[46] M. S. Lee, M. L. Wahlqvist, H. L. Yu, and W. H. Pan, "Hyperuricemia and metabolic syndrome in Taiwanese children," Asia Pacific Journal of Clinical Nutrition, vol. 16, no. 2, pp. 594-600, 2007.

[47] I. F. Shatat, R. T. Abdallah, D. J. Sas, and S. M. Hailpern, "Serum uric acid in US adolescents: distribution and relationship to demographic characteristics and cardiovascular risk factors," Pediatric Research, vol. 72, no. 1, pp. 95-100, 2012.

[48] Y. Kawasaki, M. Hosoya, S. Yasumura et al., "The basic data for residents aged 15 years or younger who received a comprehensive health check in 2011-2012 as a part of the fukushima health management survey after the great east Japan earthquake," Fukushima Journal of Medical Science, vol. 61, no. 2, pp. 101-110, 2015.

[49] N. Li, S. Zhang, W. Li et al., "Prevalence of hyperuricemia and its related risk factors among preschool children from China," Scientific Reports, vol. 7, no. 1, p. 9448, 2017.

[50] L. Tang, M. Kubota, A. Nagai, K. Mamemoto, and M. Tokuda, "Hyperuricemia in obese children and adolescents: the relationship with metabolic syndrome," Pediatric Reports, vol. 2, no. 1, p. e12, 2010.

[51] S. C. Modino, M. G. De armas, S. M. Mejías et al., "Hyperuricemia and metabolic syndrome in children with overweight and obesity," Endocrinología y Nutrición, vol. 59, no. 7, pp. 533-538, 2012.

[52] A. S. Cardoso, N. C. Gonzaga, C. C. M. Medeiros, and D. F. de Carvalho, "Association of uric acid levels with components of metabolic syndrome and non-alcoholic fatty liver disease in overweight or obese children and adolescents," Jornal de Pediatria (Versão em Português), vol. 89, no. 4, pp. 412-418, 2013.

[53] L. Ságodi, V. Fehér, E. Kiss-Tóth, A. Almási, and L. Barkai, “Az elhízás anyagcsere-szövődményei serdülőkorban, különös tekintettel az emelkedett húgysavszintek előfordulására," Orvosi Hetilap, vol. 156, no. 22, pp. 888-895, 2015.

[54] M. Gil-Campos, C. M. Aguilera, and C. A. Gil, "Uric acid is associated with features of insulin resistance syndrome in obese children at prepubertal stage," Nutricion Hospitalaria, vol. 24, no. 5, pp. 607-613, 2009.

[55] M. Krzystek-Korpacka, E. Patryn, I. Kustrzeba-Wojcicka, J. Chrzanowska, A. Gamian, and A. Noczynska, "Genderspecific association of serum uric acid with metabolic syndrome and its components in juvenile obesity," Clinical Chemistry and Laboratory Medicine, vol. 49, no. 1, pp. 129136, 2011.

[56] E. Kuwahara, Y. Murakami, T. Okamura et al., "Increased childhood BMI is associated with young adult serum uric acid levels: a linkage study from Japan," Pediatric Research, vol. 81, no. 2, pp. 293-298, 2017.

[57] H. K. Choi and E. S. Ford, "Prevalence of the metabolic syndrome in individuals with hyperuricemia," American Journal of Medicine, vol. 120, no. 5, pp. 442-447, 2007.

[58] S. Bussler, M. Penke, G. Flemming et al., "Novel insights in the metabolic syndrome in childhood and adolescence," Hormone Research in Paediatrics, vol. 88, no. 3-4, pp. 181-193, 2017.

[59] M. D. DeBoer, L. Dong, and M. J. Gurka, "Racial/ethnic and sex differences in the relationship between uric acid and metabolic syndrome in adolescents: an analysis of national health and nutrition survey 1999-2006," Metabolism, vol. 61, no. 4, pp. 554-561, 2012.

[60] H. L. Sun, D. Pei, K. H. Lue, and Y. L. Chen, "Uric acid levels can predict metabolic syndrome and hypertension in adolescents: a 10-year longitudinal study," PLoS One, vol. 10, no. 11, Article ID e0143786, 2015.

[61] D. I. Feig and R. J. Johnson, "Hyperuricemia in childhood primary hypertension," Hypertension, vol. 42, no. 3, pp. 247-252, 2003.

[62] D. P. Jones, P. A. Richey, B. S. Alpert, and R. Li, "Serum uric acid and ambulatory blood pressure in children with primary hypertension," Pediatric Research, vol. 64, no. 5, pp. 556-561, 2008.

[63] F. Viazzi, L. Antolini, M. Giussani et al., "Serum uric acid and blood pressure in children at cardiovascular risk," Pediatrics, vol. 132, no. 1, pp. e93-e99, 2013.

[64] B. Park, H. A. Lee, S. H. Lee et al., "Association between serum levels of uric acid and blood pressure tracking in childhood," American Journal of Hypertension, vol. 30, no. 7, pp. 713-718, 2017.

[65] A. B. Alper Jr., W. Chen, L. Yau, S. R. Srinivasan, G. S. Berenson, and L. L. Hamm, "Childhood uric acid predicts adult blood pressure," Hypertension, vol. 45, no. 1, pp. 34-38, 2005.

[66] M. Yanik and D. I. Feig, "Serum urate: a biomarker or treatment target in pediatric hypertension?," Current Opinion in Cardiology, vol. 28, no. 4, pp. 433-438, 2013.

[67] A. H. M. W. Islam, S. Yamashita, K. Kotani et al., "Fasting plasma insulin level is an important risk factor for the development of complications in Japanese obese childrenresults from a cross-sectional and a longitudinal study," Metabolism, vol. 44, no. 4, pp. 478-485, 1995.

[68] I. I. Sakou, T. Psaltopoulou, T. N. Sergentanis et al., "Insulin resistance and cardiometabolic risk factors in obese children and adolescents: a hierarchical approach," Journal of Pediatric Endocrinology and Metabolism, vol. 28, no. 5-6, pp. 589-596, 2015.

[69] C. Denzer, R. Muche, H. Mayer, E. Heinze, K. M. Debatin, and M. Wabitsch, "Serum uric acid levels in obese children and adolescents: linkage to testosterone levels and pre-metabolic syndrome," Journal of Pediatric Endocrinology and Metabolism, vol. 16, no. 9, pp. 1225-1232, 2003.

[70] Y. C. Ko, M. C. Huang, T. N. Wang, S. J. Chang, L. Y. Tsai, and H. P. Tu, "Prevalence and risk factors associated with dyslipidaemia in children and adolescents among ethnic groups in Taiwan," Public Health, vol. 119, no. 6, pp. 489-497, 2005.

[71] I. Narverud, K. Retterstøl, P. O. Iversen et al., "Markers of atherosclerotic development in children with familial 
hypercholesterolemia: a literature review," Atherosclerosis, vol. 235, no. 2, pp. 299-309, 2014.

[72] L. Pacifico, V. Cantisani, C. Anania et al., "Serum uric acid and its association with metabolic syndrome and carotid atherosclerosis in obese children," European Journal of Endocrinology, vol. 160, no. 1, pp. 45-52, 2009.

[73] M. Ishiro, R. Takaya, Y. Mori et al., "Association of uric acid with obesity and endothelial dysfunction in children and early adolescents," Annals of Nutrition and Metabolism, vol. 62, no. 2, pp. 169-176, 2012.

[74] S. A. Fathallah-Shaykh and M. T. Cramer, "Uric acid and the kidney," Pediatric Nephrology, vol. 29, no. 6, pp. 999-1008, 2014.

[75] K. E. Rodenbach, M. F. Schneider, S. L. Furth et al., "Hyperuricemia and progression of CKD in children and adolescents: the chronic kidney disease in children (CKiD) cohort study," American Journal of Kidney Diseases, vol. 66, no. 6, pp. 984-992, 2015.

[76] F. G. Sharbaf and F. Assadi, "Effect of allopurinol on the glomerular filtration rate of children with chronic kidney disease," Pediatric Nephrology, vol. 33, no. 8, pp. 1405-1409, 2018.

[77] S.-H. Hsia, I.-J. Chou, C.-F. Kuo et al., "Survival impact of serum uric acid levels in children and adolescents," Rheumatology International, vol. 33, no. 11, pp. 2797-2802, 2013.

[78] G. P. August, S. Caprio, I. Fennoy et al., "Prevention and treatment of pediatric obesity: an endocrine society clinical practice guideline based on expert opinion," Journal of Clinical Endocrinology \& Metabolism, vol. 93, no. 12, pp. 4576-4599, 2008.

[79] K. Togashi, H. Masuda, and K. Iguchi, "Effect of diet and exercise treatment for obese Japanese children on abdominal fat distribution," Research in Sports Medicine, vol. 18, no. 1, pp. 62-70, 2010.

[80] M. Krzystek-Korpacka, E. Patryn, I. Kustrzeba-Wojcicka, J. Chrzanowska, A. Gamian, and A. Noczynska, "The effect of a one-year weight reduction program on serum uric acid in overweight/obese children and adolescents," Clinical Chemistry and Laboratory Medicine, vol. 49, no. 5, pp. 915-921, 2011.

[81] R. J. Torres, C. Prior, and J. G. Puig, "Efficacy and safety of allopurinol in patients with hypoxanthine-guanine phosphoribosyltransferase deficiency," Metabolism, vol. 56, no. 9, pp. 1179-1186, 2007.

[82] J. Harambat, G. Bollée, M. Daudon, I. Ceballos-Picot, A. Bensman, and APRT Study Group, "Adenine phosphoribosyltransferase deficiency in children," Pediatric Nephrology, vol. 27, no. 4, pp. 571-579, 2012.

[83] G. P. Smit, "The long-term outcome of patients with glycogen storage disease type Ia," European Journal of Pediatrics, vol. 152, no. 1, pp. S52-S55, 1993.

[84] L. D. Fairbanks, J. S. Cameron, G. Venkat-Raman et al., "Early treatment with allopurinol in familial juvenile hyerpuricaemic nephropathy (FJHN) ameliorates the long-term progression of renal disease," QJM: An International Journal of Medicine, vol. 95, no. 9, pp. 597-607, 2002.

[85] B. Soletsky and D. I. Feig, "Uric acid reduction rectifies prehypertension in obese adolescents," Hypertension, vol. 60, no. 5, pp. 1148-1156, 2012.

[86] F. Assadi, “Allopurinol enhances the blood pressure lowering effect of enalapril in children with hyperuricemic essential hypertension," Journal of Nephrology, vol. 27, no. 1, pp. 51-56, 2014.
[87] R. Somkrua, E. E. Eickman, S. Saokaew, M. Lohitnavy, and N. Chaiyakunapruk, "Association of HLA-B $* 5801$ allele and allopurinol-induced stevens johnson syndrome and toxic epidermal necrolysis: a systematic review and meta-analysis," BMC Medical Genetics, vol. 12, no. 1, p. 118, 2011.

[88] M. A. Becker, J. Kisicki, R. Khosravan et al., "Febuxostat (TMX-67), a novel, non-purine, selective inhibitor of xanthine oxidase, is safe and decreases serum urate in healthy volunteers," Nucleosides, Nucleotides and Nucleic Acids, vol. 23, no. 8-9, pp. 1111-1116, 2004.

[89] Y. Kaku and M. Nishimura, "Febuxostat for hyperuricemia of CKD children," Pediatric Nephrology, vol. 31, p. 1907, 2016.

[90] K. Kishimoto, R. Kobayashi, D. Hori, H. Sano, D. Suzuki, and K. Kobayashi, "Febuxostat as a prophylaxis for tumor lysis syndrome in children with hematological malignancies," Anticancer Research, vol. 37, no. 10, pp. 5845-5849, 2017.

[91] C.-H. Pui, S. Jeha, D. Irwin, and B. Camitta, "Recombinant urate oxidase (rasburicase) in the prevention and treatment of malignancy-associated hyperuricemia in pediatric and adult patients: results of a compassionate-use trial," Leukemia, vol. 15, no. 10, pp. 1505-1509, 2001.

[92] B. Coiffier, A. Altman, C.-H. Pui, A. Younes, and M. S. Cairo, "Guidelines for the management of pediatric and adult tumor lysis syndrome: an evidence-based review," Journal of Clinical Oncology, vol. 26, no. 16, pp. 2767-2778, 2008.

[93] D. K. Cheuk, A. K. Chiang, G. C. Chan, and S. Y. Ha, "Urate oxidase for the prevention and treatment of tumor lysis syndrome in children with cancer," Cochrane Database of Systematic Reviews, vol. 16, no. 6, article CD006945, 2017. 


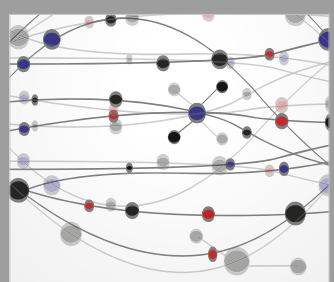

The Scientific World Journal
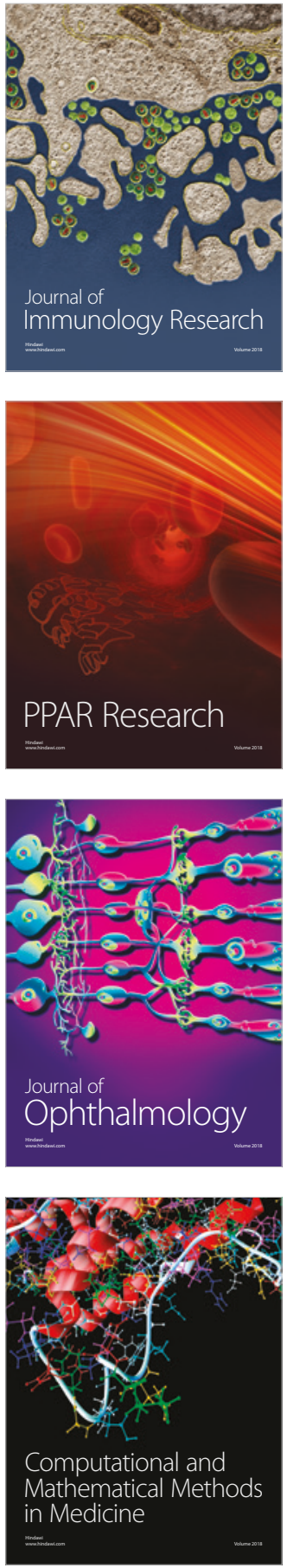

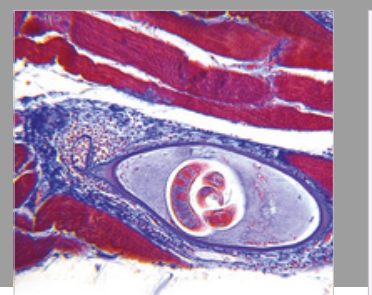

Gastroenterology Research and Practice

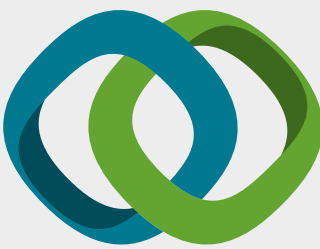

\section{Hindawi}

Submit your manuscripts at

www.hindawi.com
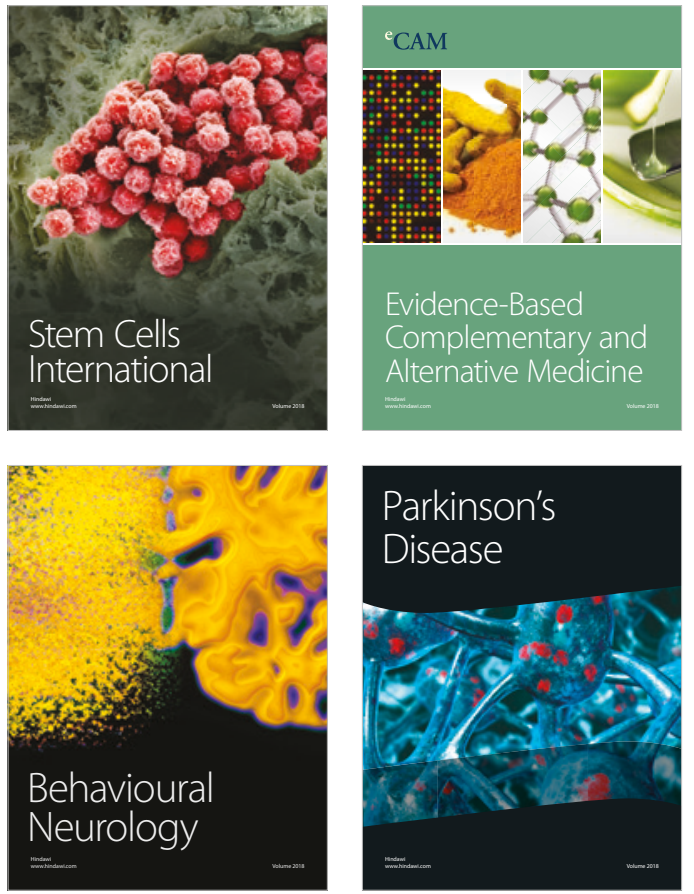

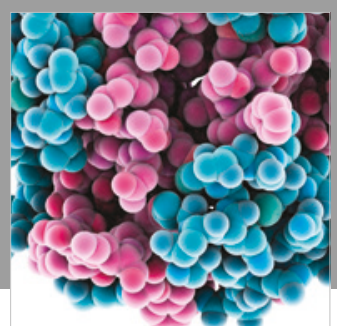

ournal of

Diabetes Research

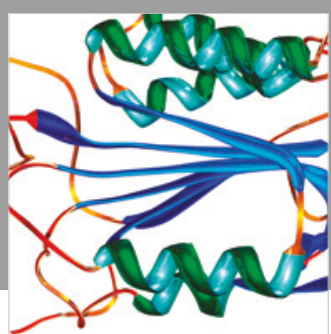

Disease Markers
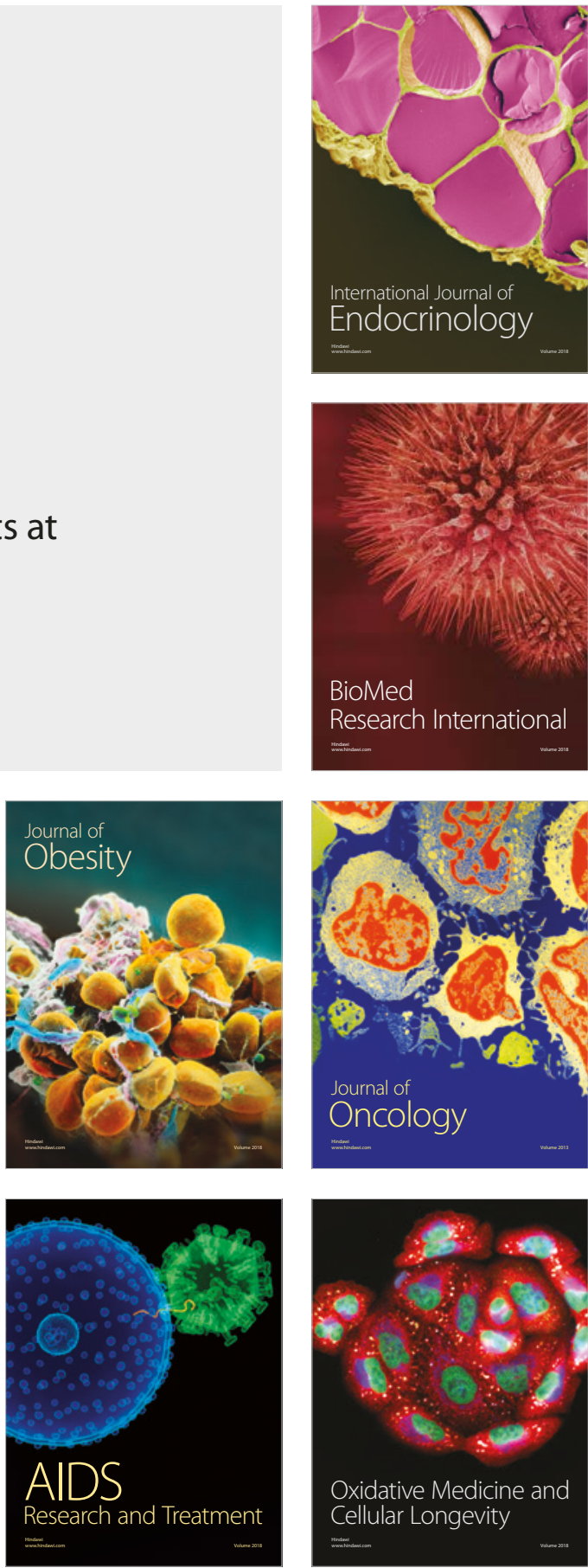